\title{
IJCARS: MICCAI 2019 special issue
}

\author{
Terry Peters $^{1} \cdot$ Caroline Essert $^{2} \cdot$ Ali Khan ${ }^{1}$
}

Published online: 21 October 2020

(c) CARS 2020

It is with great pleasure that we present this IJCARS-MICCAI special issue, highlighting contributions relating to Image-Guided Interventions presented at MICCAI 2019.

As Program Co-chairs for the 2019 MICCAI meeting in Shenzhen China, we also took on the role of Guest Editors for this issue of IJCARS, selecting the best CAI contributions to MICCAI, and inviting the authors to submit revised and extended accounts of their work to IJCARS.

The quality of MICCAI submitted manuscripts is already at a high standard, and the multi-level double-blind peerreview process, which accepts around $30 \%$ of the submitted papers, ensured that those accepted for the conference are of excellent quality. The MICCAI review process consists of an initial review by at least three reviewers, followed by discussions between reviewers and Area Chairs. Papers are then accepted or rejected outright or authors are asked to provide a rebuttal to reviewers' comments. Approximately half of the papers providing a rebuttal were finally accepted for inclusion in the conference.

For MICCAI 2019, we received 1809 full submissions which were sent out for peer review. Of these, $63 \%$ were considered as pure Medical Image Computing (MIC), and 37\% contained material relevant to the CAI community. Topics related to CAI covered the following themes: Surgical and Interventional Simulation Systems; Medical Robotics and Haptics; Image-Guided Interventions and Surgery ; Interven- tional Imaging Systems; Interventional Software and User Interfaces; Interventional Tracking and Navigation; Surgical Data Science; Surgical Planning and Simulation; Surgical Skill and Work Flow Analysis; Surgical Visualization and Mixed, Augmented and Virtual Reality; and Visualization in Biomedical Imaging.

Based on their scoring in the initial review process, we invited 25 submissions from the CAI-related group to be substantially revised and updated (less than $20 \%$ overlap in text, new figures and new data) for this IJCARS special issue. Fifteen authors responded positively to this invitation.

The new manuscripts were then submitted to IJCARS as new submissions, which were then subjected to the normal rigorous IJCARS review process, that included at least three reviewers and the opportunity to improve the manuscript in response to their comments.

We would like to take this opportunity to thank the Area Chairs, and all of the reviewers of the initial MICCAI submissions, as well as those who took part in the review process for this special issue of IJCARS. We would also like to thank the management of IJCARS, particularly Heinz Lemke and Franziska Schweikert, for their continuing high standards and efficiency in ensuring both the high quality of this special issue and that it appeared in a timely fashion.

Publisher's Note Springer Nature remains neutral with regard to jurisdictional claims in published maps and institutional affiliations.

Terry Peters

tpeters@ robarts.ca

Caroline Essert

essert@unistra.fr

Ali Khan

alik@robarts.ca

1 Robarts Research Institute, Western University, 1151

Richmond St, London, ON N6A 3K7, Canada

2 ICube, Université de Strasbourg, 300, Boulevard Sebastien Brant, 67400 Illkirch, Strasbourg, France 